\title{
DETERMINATION OF CRACK INITIATION AND PROPAGATION IN TWO DISC SHAPED SPECIMENS USING THE IMPROVED MAXIMUM TANGENTIAL STRESS CRITERION
}

\author{
Mosleh Eftekhari \\ Department of Mining Engineering, Science and Research Branch, Islamic Azad University, Tehran, Iran \\ e-mail: eftekhari_mosleh@yahoo.com; mosleh.eftekhari@mi.iut.ac.ir
}

Alireza Baghbanan, Ehsan Mohtarami

Department of Mining Engineering; Isfahan University of Technology (IUT), Isfahan, Iran

HAMid HaShemolhosseini

Department of Civil Engineering; Isfahan University of Technology (IUT), Isfahan, Iran

\begin{abstract}
The crack initiation angle and propagation path for two different disc shaped test specimens (i.e., SCB and CBD specimen) are investigated experimentally and theoretically. The Maximum Tangential Stress (MTS) criterion does not calculate the crack initiation angle in SCB and CBD specimens correctly. Moreover, at the angles after occurrence of pure mode II, where the stress intensity factor of mode I becomes negative, this criterion is not applicable. Therefore, in this research work, Improved MTS (IMTS) criterion which has been implemented in the extended finite element method and is applicable under tensile and compressive loading conditions to examine the crack propagation path in the aforementioned disc shaped specimens. Furthermore, an experimental study on a cracked Brazilian disc specimen has been conducted at different angles. Results of IMTS criterion in these specimens show that the crack propagation path and the crack initiation angle can be predicted theoretically by using IMTS criterion.
\end{abstract}

Keywords: Extended Finite Element Method (XFEM), maximum tangential stress (MTS) criterion, improved maximum tangential stress (IMTS) criterion, crack propagation, stress intensity factor

\section{Introduction}

Investigation of the fracture grow path is an important task to optimize size of rock pieces or control stability of cracked rock structures in many applications of rock engineering such as rock cuttings, excavations and rock stability analysis. In rock structures, most of failures occur under a mixed mode in practice (combination of the opening and shearing mode), and thus fracture path of cracked structures may grow in curved paths and not necessarily along the direction of the initial crack (Aliha et al., 2010). Therefore, investigating the crack initiation angle and the crack propagation path under the mixed loading mode are favorite subjects for researchers in the field of rock mechanics. There are a large number of theoretical models (Erdogan and Sih, 1963; Hussain et al., 1974; Nuismer, 1975; Palaniswamy and Knauss, 1972; Sih, 1974; Theocaris and Andrianopoulos, 1982) and experimental techniques (Grassl and Rempling, 2007; Isaksson and Ståhle, 2002; Lin et al., 2009; Song et al., 2004; Xeidakis et al., 1997) for investigating the mixed mode of crack growth in rock materials. Theoretical failure criteria such as the Maximum Tangential Stress (MTS) criterion (Erdogan and Sih, 1963), the minimum strain energy density criterion (Sih, 1974), and the maximum energy release rate criterion (Hussain et al., 1974) have been frequently used by researchers working on rock and geo-material fields in order to estimate 
the direction of the mixed mode in the crack growth process. These failure models are usually developed based on stress, strain, and energy conditions in front of the crack tip, and the stress intensity factor is often used to estimate the crack growth direction.

Various numerical methods such as the boundary element method, finite difference method, meshless method and finite element method (FEM) are adopted to problem-solving in fracture mechanics. The common purpose of these methods has always been to achieve a precise, straightforward and a developed practical solution containing advantages of previous methods and upcoming their restrictions and shortcomings. This process of development of numerical methods led to the appearance of a novel method in 1999, called the extended finite element method (XFEM) which is based on the finite element method. This method takes advantage of all features of the finite element method in solving different problems, and it has resolved its shortcomings in predicting of crack initiation and propagation. In this method, the crack is defined as mesh independent, hence, the crack is able to grow anywhere through the finite element mesh. Extensive studies using this method which have been conducted in a short time after its appearance indicate that this method has a great capability in modeling problems associated with fracture mechanics (Budyn et al., 2004; Campilho et al., 2011; Fan and Jing, 2013; Huynh and Belytschko, 2009; Mergheim et al., 2005; Singh et al., 2012). Thus this method has been chosen as the numerical solution tool in our research work.

The Maximum Tangential Stress criterion in which the crack is propagated from the crack tip along the direction of maximum tangential stress has been used more extensively in the XFEM for the modeling of crack growth. The direction of crack propagation when the MTS criterion is assigned in numerical modeling depends on the calculated value of stress intensity factors as follows

$$
\theta_{c}=2 \tan ^{-1} \frac{1}{4}\left(\frac{K_{\mathrm{I}}}{K_{\mathrm{II}}} \pm \sqrt{\left(\frac{K_{\mathrm{I}}}{K_{I I}}\right)^{2}+8}\right)
$$

where $\theta_{c}$ is the crack initiation angle and $K_{\mathrm{I}}$ and $K_{\mathrm{II}}$ are stress intensity factors in modes I and II, respectively. When the crack is placed in a compressive stress field, the value of the stress intensity factor of mode I becomes negative and this criterion is no longer able to estimate the direction of crack propagation. While, rocks unlike most engineering materials often experience compressive loading and therefore most rock failures are also occurred under such conditions. Therefore, attempts have been made to study the initiation and propagation of cracks under compressive loading conditions in the literature. Lack of efficiency of the MTS criterion under compressive conditions has been addressed by other researchers (Al-Shayea, 2005; Aliha et al., 2010; Bobet, 1997; Shen and Stephansson, 1994; Wu and Wong, 2012). Bobet (1997) compared results of crack initiation angle by MTS criterion with results obtained from uniaxial compressive tests and stated that this criterion did not offer a suitable estimation of the crack initiation angle. He also represented a stress-based criterion in order to estimate crack initiation angle on the boundary element method. Shen and Stephansson (1994) also developed the F criterion or the modified G criterion for the same purpose. Wu and Wong (2012) examined crack propagation under compressive loading using the Mohr-Coulomb criterion using the numerical manifold method.

Recently, the MTS criterion has been improved and implemented in XFEM in order to be utilized in different loading conditions from tensile to compressive loading (Eftekhari et al., 2016). This criterion is called the Improved MTS (IMTS) criterion and it has been evaluated in some examples and then used in a cracked specimen under uniaxial compression. Results from the IMTS criterion are perfectly matched with the experimental and numerical modeling results reported in the literature (Eftekhari et al., 2016).

Different laboratory specimens and experimental methods have been proposed and used to determine rock fracture toughness so far. However, a review of previous studies indicates that the 
most commonly used specimens for conducting fracture tests and studying crack growth in rocks and geo-materials are disc shaped specimens such as Cracked Brazilian Disc (CBD) (Atkinson et al., 1982; Awaji and Sato, 1978; Eftekhari et al., 2015a), Hollow Centre Cracked Disc (HCCD) (Choi et al., 1988; Eftekhari et al., 2015c; Shiryaev and Kotkis, 1983), Flattened Brazilian Disc (Wang and Xing, 1999; Wang et al., 2004), Modified Ring (Thiercelin, 1989; Thiercelin and Roegiers, 1986), Semi-Circular Bend (SCB) specimen (Chong et al., 1987; Eftekhari et al., 2015b; Kuruppu and Chong, 2012).

Two frequently employed disc type specimens are the cracked Brazilian disc subjected to diametral compression and the semicircular bend specimen subjected to three-point bend loading. Simple geometry, straight forward preparation and easy loading and also capability to perform different mode combinations are the main advantages of these specimens. Hence, these test specimens have been used frequently to investigate mixed mode crack growth of rock materials (Al-Shayea, 2005; Ayatollahi and Aliha, 2008; Chen et al., 1998; Eftekhari et al., 2015a; Eftekhari et al., 2015b; Liu et al., 2007). Recently, International Society for Rock Mechanics (ISRM) has suggested the SCB specimen to be the new standard specimen in the determination of the fracture toughness of pure mode I (Kuruppu et al., 2015).

Figure 1 shows the geometry and loading condition of the CBD and SCB specimens used for mixed mode I/II fracture tests. In the CBD specimen, the angle $\beta$ is defined as an angle between the direction of centre crack with length $2 a$ and the applied loading direction. The SCB specimen is a semicircular disc of radius $R$ which has an angled edge crack with respect to the applied load $P$. For both specimens, pure modes I and II and the mixed mode can be provided by changing the crack angle $\beta$. Obviously, $\beta=0^{\circ}$ is associated with the case of pure mode I condition. By increasing the loading angle from zero, mode II is introduced.
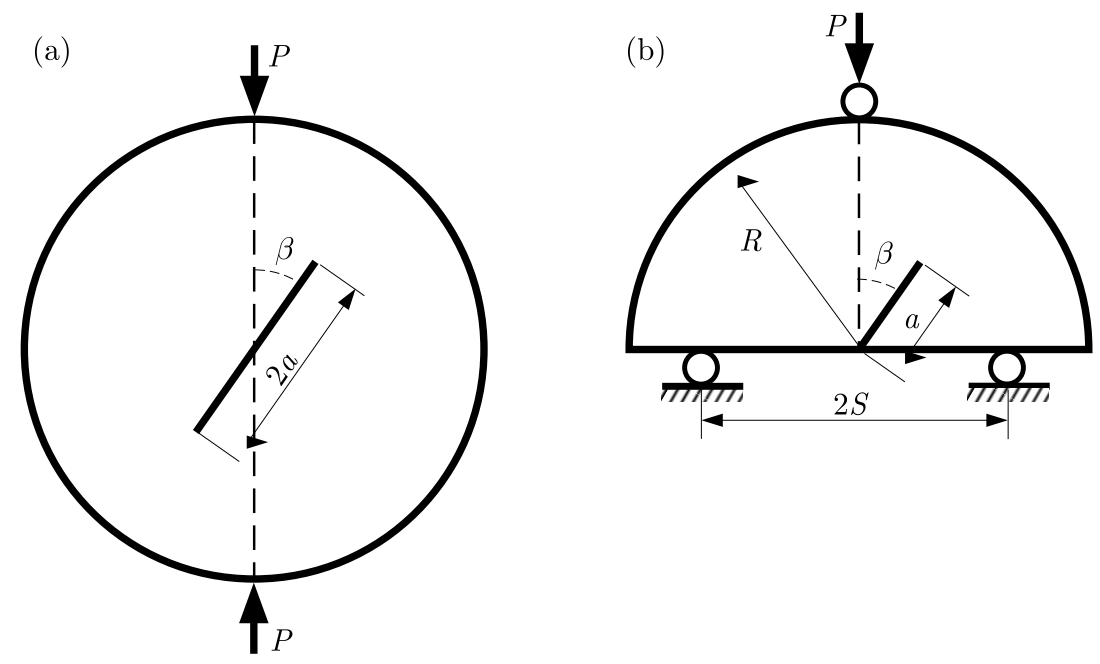

Fig. 1. Geometry and loading conditions of CBD and SCB specimens subjected to mixed mode I/II loading: (a) CBD specimen, (b) SCB specimen

Experimental results with $\mathrm{CBD}$ and SCB specimens have showed that the approximated crack initiation angle by the MTS criterion is different with the measured values by laboratory experiments (Aliha et al., 2010). Aliha et al. (2010) introduced a criterion called Generalized MTS (GMTS) in order to estimate the crack propagation direction more accurately.

Theoretically, when $K_{\mathrm{I}}$ value is equal to zero, pure mode II occurs. In the SCB and CBD specimens, $K_{\mathrm{I}}$ value decreases by increasing the initial crack angle, and after the angle of occurrence of pure mode II, its value becomes negative (Eftekhari et al., 2015a; Eftekhari et al., 2015b). Results of investigations of crack initiation and propagation in the CBD specimen shows that the angle of appearance pure mode II happens at lower than $30^{\circ}$ (Eftekhari et al., 2015a), 
thus, at the angles beyond this value, the stress intensity factor of mode I becomes negative and the MTS and GMTS criteria which are based on the stress intensity factor are no longer applicable. However, experimental results by this specimen have showed that at the crack angles higher than this value, fracture and crack propagation path occurs similar to the mixed mode (Al-Shayea, 2005; Ghazvinian et al., 2013; Haeri et al., 2014). This phenomenon was observed and reported in Eftekhari et al. (2015c), when they showed that the fracturing mode in macro and micro scales are different. The comparison result indicates that the fracture in micro-scale, unlike the macro-scale, includes a combination of different modes of fracturing (Eftekhari et al., 2015c). Therefore, as also stated by Sharafisafa and Nazem (2014), it seems that crack propagation under compressive conditions is more controlled by tensile stresses around the crack tip.

In this research work, a numerical sensitivity analysis about crack propagation in SCB and CBD specimens with different initial crack angles when the IMTS criterion is assigned, has been conducted. The results have been also compared with laboratory tests and the reported results in the literature.

\section{Extended Finite Element Method (XFEM) and Improved MTS (IMTS) criterion}

Extended Finite Element Method (XFEM) was initially introduced by Belytschko and Black (1999). They presented a method based on the finite element method for modeling of crack propagation which does not need the re-meshing process. In this method, discontinuous enriched functions are added to the finite element approximation for crack demonstration and the crack grows arbitrarily within the finite element mesh. The most important and effective step toward improvement of the extended finite element method was taken by (Moës et al., 1999) and (Dolbow, 1999). The existence of a crack in the extended finite element method leads to two different enrichments in the problem; crack interior and crack tip enrichment. The crack interior is enriched by a modified discontinuous Heaviside function (Eq. (2.1)), and the crack tip is enriched using functions presented in Eq. (2.2)

$$
H(x)=\left\{\begin{array}{lll}
+1 & \text { for } \quad x>0 \\
-1 & \text { for } \quad x<0
\end{array}\right.
$$

and

$$
\left\{F_{j}(r, \text { thet } a)\right\}_{j=1,2,3,4}=\left\{\sqrt{r} \sin \frac{\theta}{2}, \sqrt{r} \cos \frac{\theta}{2}, \sqrt{r} \sin \frac{\theta}{2} \sin \theta, \sqrt{r} \cos \frac{\theta}{2} \sin \theta\right\}
$$

Among these functions, only the first function $(\sqrt{r} \sin (\theta / 2))$ is discontinuous, which is indicative of the function discontinuity along two faces of the crack. The extended finite element approximation for the displacement field is defined as follows

$$
u^{h}(x)=\sum_{i \in I} N_{i}(x) u_{i}+\sum_{j \in J} N_{j}(x) H_{j}(x) a_{j}+\sum_{k \in K} N_{k}(x) \sum_{l=1}^{4} F_{l}(x) b_{k}^{l}
$$

where $N(x)$ are shape functions, $u_{i}$ are nodal displacements (standard degrees of freedom), $a_{j}$ are vectors of additional degrees of nodal freedom associated with the Heaviside function, and $b_{k}^{l}$ are vectors of additional degrees of nodal freedom associated with the elastic asymptotic cracktip functions. In equation (2.3), $I$ is the set of all nodes in the mesh, $J$ is the set of enriched nodes with discontinuous enrichment and $K$ is the set of nodes enriched with asymptotic enrichment. 
All nodes of the element intersected by the crack are enriched by the step function, and also the nodes of element containing a crack tip are enriched by the near-tip displacement field. Equations and matrixes in the extended finite element method proceed similar to the conventional finite element method. The displacement field approximation space in form of the extended finite element (Eq. (2.3)) is replaced in equilibrium equations. From this enriched approximation, the standard discrete equation $\mathbf{K d}=\mathbf{f}$ is obtained, where $\mathbf{d}$ is the vector of unknowns or degrees of freedom (for both common finite element degrees and additional degrees of freedom associated with enrichment), $\mathbf{f}$ is the external vector of forces and $\mathbf{K}$ is the stiffness matrix. The vectors and matrices are generally obtained by assemblage in each element.

A numerical code called MEX-FEM has been developed based on XFEM formulation with $\mathrm{C}++$ programming language and employed into simulation of fracture propagation in some specimens of rock such as the cracked Brazilian disc (Eftekhari et al., 2014, 2015a) and the semi-circular bend specimen (Eftekhari et al., 2015b). In order to determine the direction of crack propagation, the Improved MTS criterion which was previously introduced and verified by the authors and reported in Eftekhari et al. (2016), is employed. A tension crack is propagated from the tip of a pre-existing crack in the direction of the maximum tangential tensile stress as also previously employed by Bobet for the modeling of crack propagation by the Boundary Element Method (Bobet and Einstein, 1998) and formulated as follows

$$
\frac{\partial \sigma_{\theta}}{\partial \theta}=0 \quad \frac{\partial^{2} \sigma_{\theta}}{\partial \theta^{2}}<0
$$

The direction of crack propagation with the IMTS criterion is similar to the MTS criterion, while the initiation angle is determined regarding the values of stress near the crack tip and it is completely independent of the value of the SIF. In this case, the SIF value does not compute by the interaction integral procedure. In order to find the direction of maximum tangential stress, the stress state near the crack tip is processed in two steps. Firstly, the values of stresses in the numerical integration points near the crack tip are transformed from the Cartesian system to the polar coordinate system in order to calculate the value of tangential stress. Since the numerical integration points are irregularly located near the crack tip, in the second step, the values of tangential stress in the proposed points with regular intervals in different angles are calculated by an interpolating process. In this way, the direction of maximum tangential stress is identified (Eftekhari et al., 2016).

\section{Simulation of SCB specimen using IMTS criterion}

Observation of crack initiation angle on the SCB specimen in laboratory shows higher values compared to the numerical simulation values by the MTS criterion (Aliha et al., 2010). Aliha et al. (2010) introduced a criterion called GMTS in order to estimate the crack propagation direction which, in this criterion, tangential stress is defined as the following expansion series and it estimates the crack initiation angle more accurate than the MTS criterion. This criterion includes a quantity called $T$ stress in addition to stress intensity factors. This stress is a non-singular and constant stress, and other values of the series are represented by $O(\sqrt{r})$ and are negligible.

$$
\sigma_{\theta \theta}=\frac{1}{\sqrt{2 \pi r}} \cos \frac{\theta}{2}\left(K_{\mathrm{I}} \cos ^{2} \frac{\theta}{2}-\frac{3}{2} K_{\mathrm{II}} \sin \theta\right)+T \sin ^{2} \theta+O(\sqrt{r})
$$

The experimental results and those calculated by the GMTS criterion in the SCB specimen with the span ratio $(S / R)$ and crack length ratio $(a / R)$ of 0.43 and 0.3 , respectively, are well matched (Aliha et al., 2010). Thus, to examine this result with the IMTS criterion, simulations 
with the span ratio $(S / R)$ of 0.43 and crack length ratio $(a / R)$ of 0.3 and the initial crack angles of $0,15,30,45$ and 60 are conducted. The results of crack initiation angle in the SCB specimen with the IMTS criterion and also experimental results and GMTS criterion as well as values calculated by the MTS criterion is demonstrated in Fig. 2.

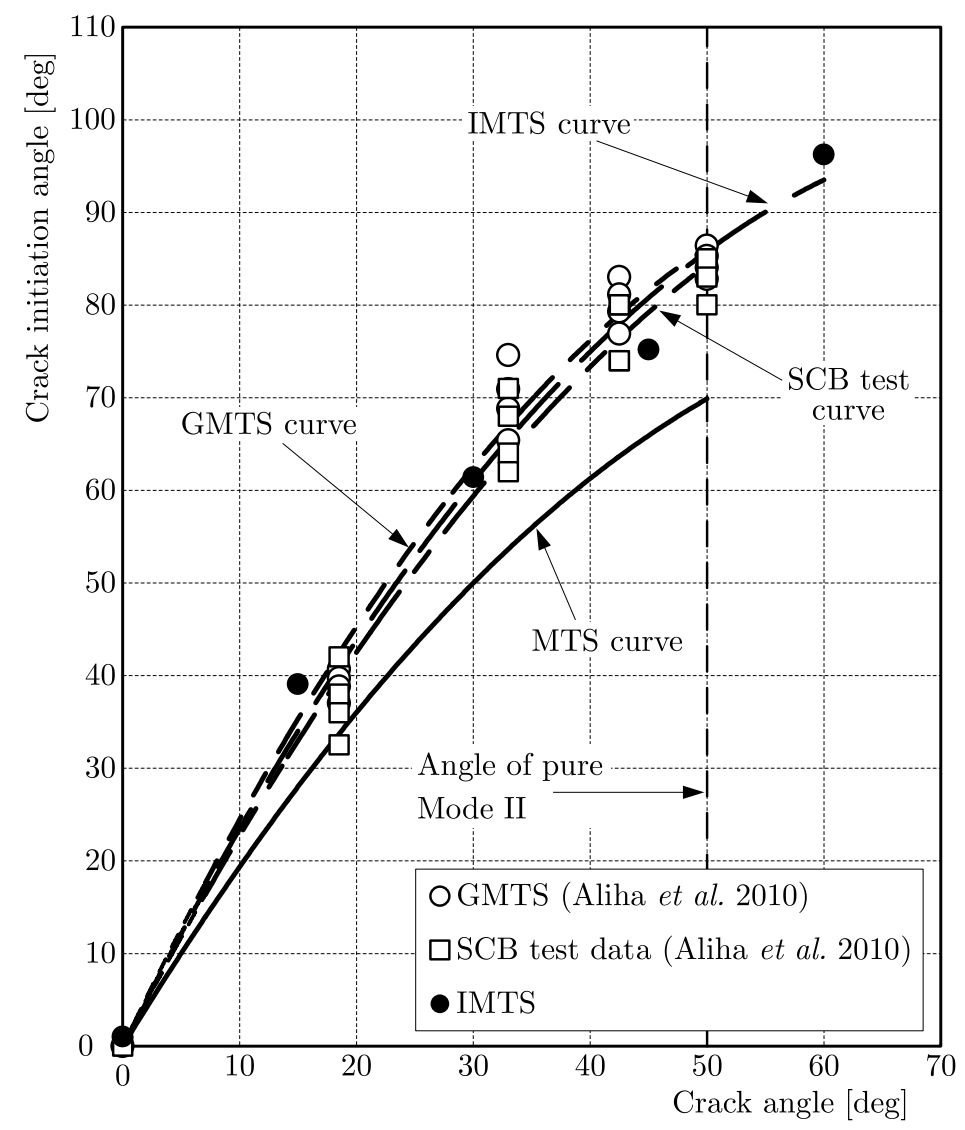

Fig. 2. Crack initiation angle in SCB specimen in laboratory and also simulation results using IMTS, GMTS and MTS criteria

It can be seen that the simulation results by the IMTS and GMTS criteria are well fitted with laboratory experimental results. In such a geometry of the SCB specimen, pure mode II occurs at angle of $50^{\circ}$. Obviously, for the crack angle beyond this value, the stress intensity factor of mode I becomes negative. Since GMTS and also MTS criteria are based on the values of stress intensity factors, investigation of the crack propagation direction at angles higher than $50^{\circ}$ are not possible in this specimen. Meanwhile, the crack initiation angle at the initial crack angle of $60^{\circ}$ is calculated using the IMTS criterion.

\section{Simulation of CBD specimen using IMTS criterion}

In contrary to the reported results about crack propagation in the SCB specimen, laboratory experimental results in the CBD specimen showed a lower approximation compared to the simulation results by the MTS criterion (Aliha et al., 2010). Although the GMTS criterion gives an acceptable estimation of the value of crack initiation angle, as mentioned earlier, the MTS and GMTS criteria are applicable up to the initial crack angle in which pure mode II occurs. On the other hand, the angle of pure mode II in the CBD specimen happens when the initial crack angle is smaller than $30^{\circ}$. While the reported experimental results in the literature show 
that the crack is propagated even at an angle larger than $30^{\circ}$ (up to $60^{\circ}$ ), just like in the mixed mode (Al-Shayea, 2005; Ghazvinian et al., 2013; Haeri et al., 2014).

Due to the inaccessibility of results of the effect of the crack initiation angle on crack propagation path in the CBD specimen, in this research work, laboratory experiments are conducted with the initial crack angles of $0^{\circ}, 15^{\circ}, 30^{\circ}, 45^{\circ}$ and $60^{\circ}$. A dental plaster with tensile and uniaxial compressive strength of 3.8 and $30.15 \mathrm{MPa}$, respectively, and density of $1.81 \mathrm{~kg} / \mathrm{cm}^{3}$ is used to prepare the CBD specimens. After weighting the plaster and mixing it with ocher powder (red color), $30 \mathrm{cc}$ per 100 grams of the plaster according to the manufacturer's recommendation is added. Its initial holding time is about 15 minutes and within 7 days the maximum strength is achieved (Babanouri et al., 2011).

Although the CBD specimen has been widely used in the modeling and laboratory experiments of rock fracture mechanics, it has not been proposed as a standard specimen by ISRM. Thus, there is no a specific and unique standard for this test. In this research work, an attempt has been made to use conditions and terms considered by other researchers in their experiments (Aliha et al., 2010; Ghazvinian et al., 2013; Haeri et al., 2014), in preparing and conducting test specimens. The experimental and numerical investigations performed by Aliha et al. (2010) on the CBD specimens with different dimensions but with the same crack length ratios show that dimensions of the specimen does not affect the results. While the crack length ratio plays the crucial role in the final results. Thus, the same proposed crack length ratio used in the literature is employed in the current study. Diameter and thickness of the disc are $60 \mathrm{~mm}$ and $25 \mathrm{~mm}$, respectively, and the crack length is $18 \mathrm{~mm}$ with the crack length ratio of 0.3 .

According to the ISRM standard for the SCB specimen (Kuruppu et al., 2015), crack thickness on the specimen should be smaller than $1.5 \mathrm{~mm}$. In this research work, in order to create a crack in the specimen, a plastic film with thickness of $1 \mathrm{~mm}$ is used. The artificial crack is also kept in the middle part of the specimen.

(a)

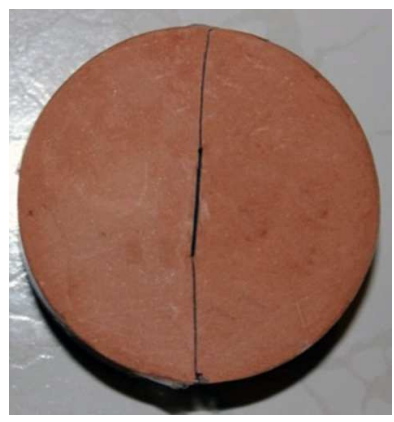

(d)

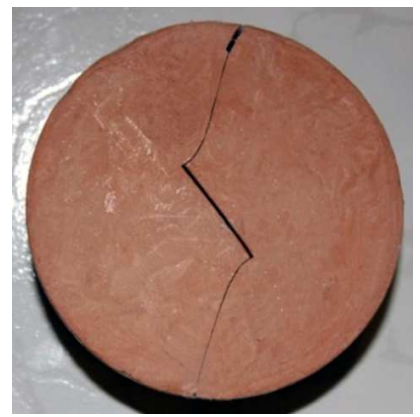

(c)

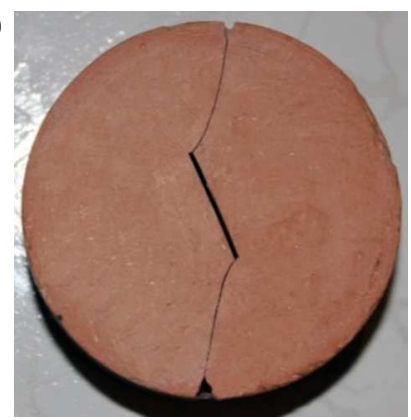

(e)

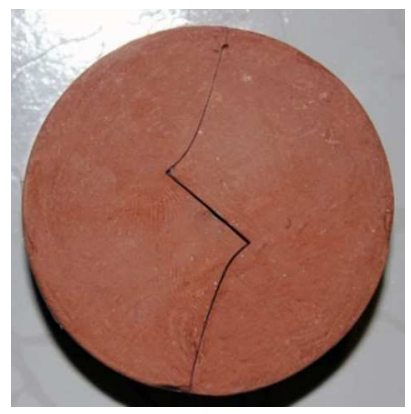

Fig. 3. Patterns of crack propagation in CBD specimen in laboratory with crack angles of (a) $0^{\circ}$, (b) $15^{\circ}$ (c) $30^{\circ}$, (d) $45^{\circ}$, (e) $60^{\circ}$

All prepared specimens, according to the aforementioned angles experience a constant compressive loading conditions. All tests are also conducted according to the ISRM standard method 
for the CCNBD specimen with rigid and parallel loading plates (Fowell, 1995). Furthermore, the loading rate was kept at $0.5 \mathrm{MPa} / \mathrm{s}$ as considered in the previous studies with the CBD specimen (Ghazvinian et al., 2013; Haeri et al., 2014). The patterns of crack propagation in the CBD specimen with different crack angles in laboratory are shown in Fig. 3.

The results show that in the case of the initial crack angle of $0^{\circ}$, the crack is propagated from its tip toward its initial direction. While at other angles, the crack is deviated from its direction and propagates toward loading points. Also it can be seen that the crack initiation angle is completely different from the results of the MTS criterion, which was presented earlier. The results of the crack initiation angle with the direction of the initial crack angle in the laboratory and also IMTS, MTS and GMTS criteria are shown in Fig. 4.

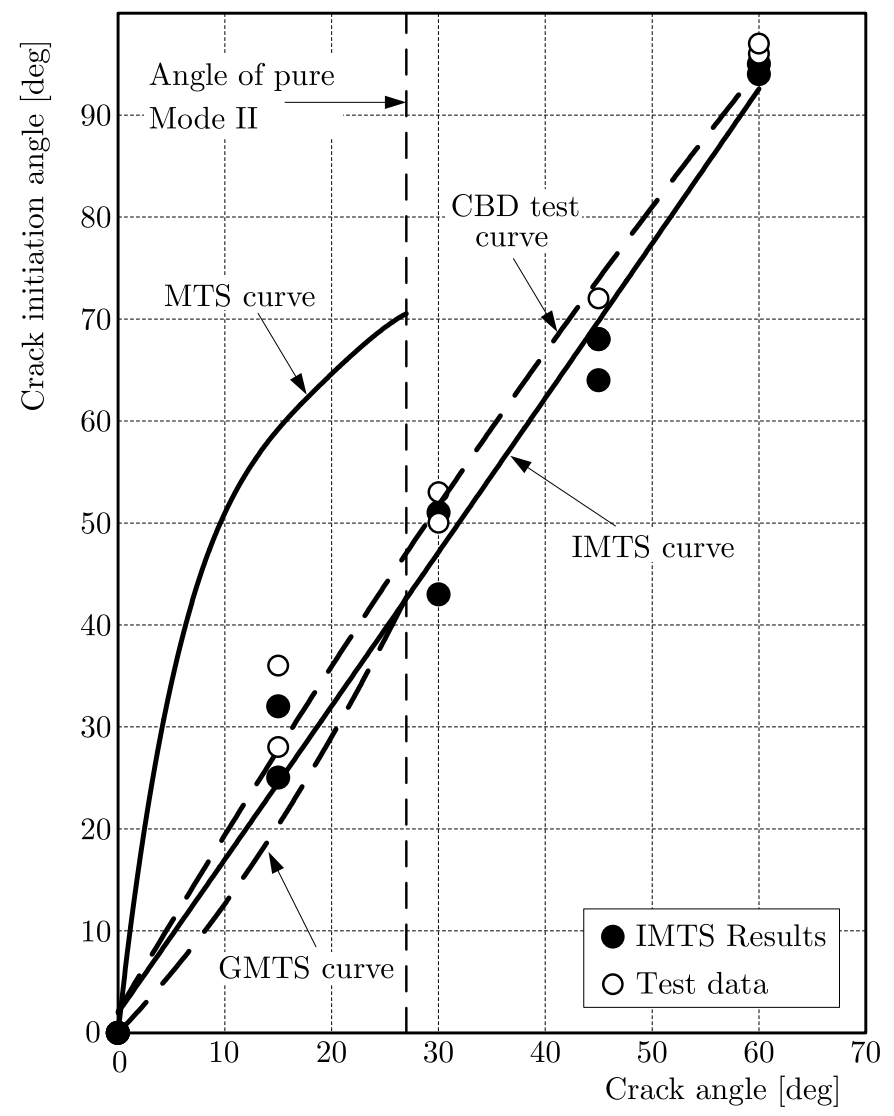

Fig. 4. Crack initiation angle in CBD specimen in laboratory and IMTS, GMTS and MTS criteria

It can be seen that the predicted values by the IMTS criterion are well matched with the laboratory experimental results and also GMTS simulation results, and completely differ from the calculated values by the MTS criterion. The GMTS and MTS criteria are applicable up to the angle of occurrence of pure mode II while in the IMTS criterion, prediction is also possible at angles beyond the predicted angle of pure mode II. The results of the crack propagation path in the specimens with different angles using the IMTS criterion are shown in Fig. 5.

The results of the crack propagation path by the IMTS criterion show that just like in the experimental specimens, the crack grows along its initial direction at the initial crack angle of zero, while at other angles, it deviates from its direction and propagates toward the loading points. By increasing the initial crack angle, the crack initiation angle is increased. In order to perform a more precise comparison, the results of the crack propagation path in the experimental specimens and also numerical simulations are shown in Fig. 6 . The crack propagation path in the experimental specimen and numerical simulation is displayed by bold and dash lines, respectively. 
(a)

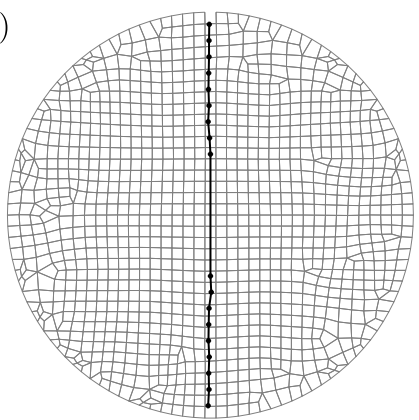

(b)

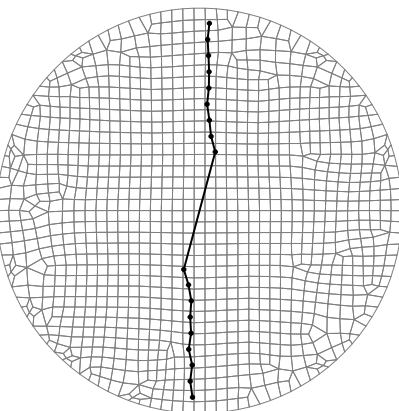

(d)

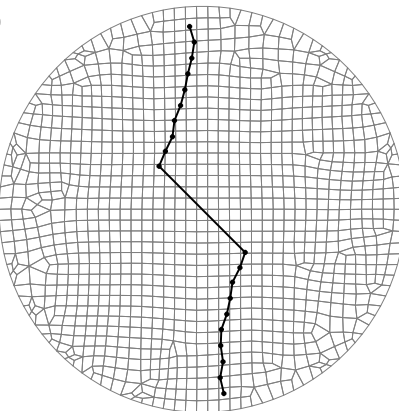

(c)

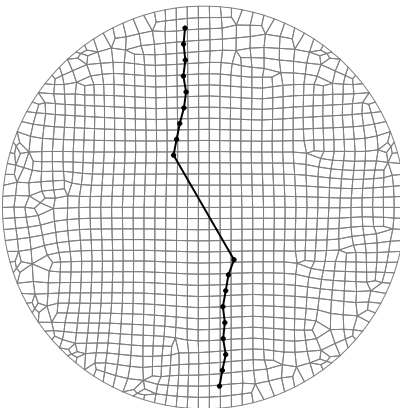

(e)

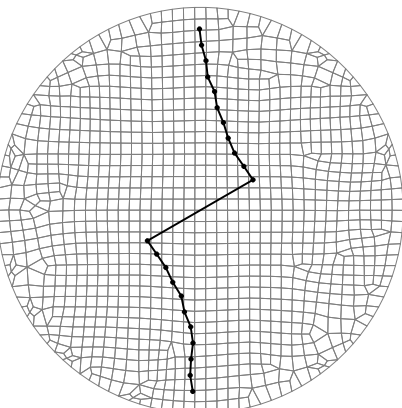

Fig. 5. Patterns of crack propagation in CBD specimen using IMTS criterion with crack angles of (a) $0^{\circ}$, (b) $15^{\circ}$ (c) $30^{\circ}$, (d) $45^{\circ}$, (e) $60^{\circ}$

(a)

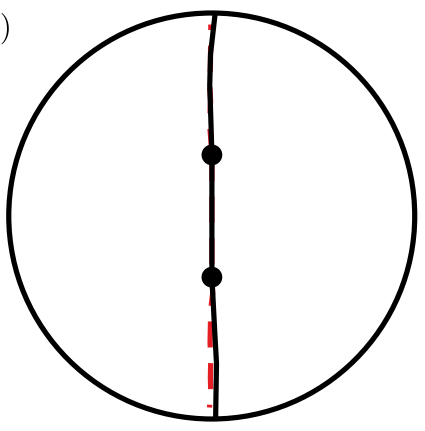

(b)

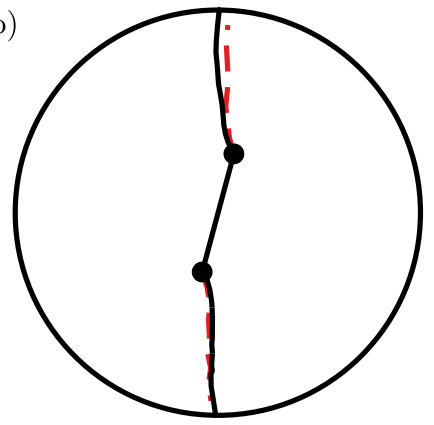

(d)

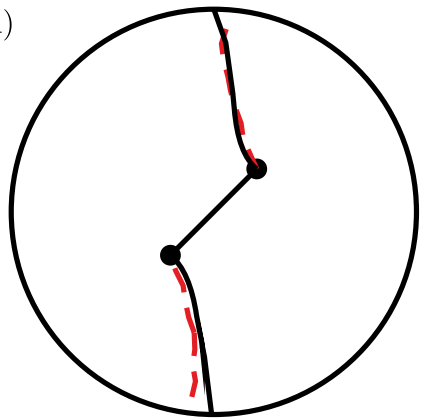

(c)

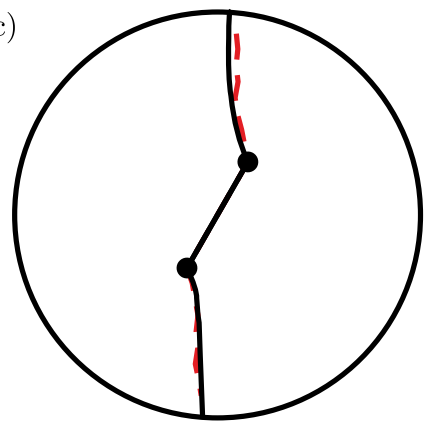

(e)

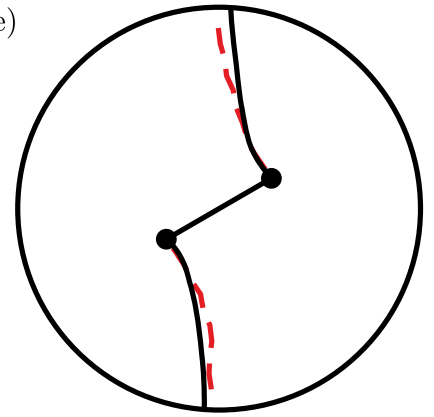

Fig. 6. Comparison of the results of the crack propagation path in experimental specimens with IMTS criterion in CBD specimen with the initial crack angle of (a) $0^{\circ}$, (b) $15^{\circ}$ (c) $30^{\circ}$, (d) $45^{\circ}$, (e) $60^{\circ}$

According to the figure, it can be seen that there is a good agreement between the results of the crack propagation path using the IMTS criterion and the experimental results.

The results of application of the IMTS criterion in XFEM to predict the crack propagation path in different specimens show that this criterion can precisely calculate the crack propagation direction in a cracked specimen under tension or compression. 


\section{Conclusion}

Maximum Tangential Stress (MTS) criterion does not calculate the crack initiation angle in SCB and CBD specimens correctly. Moreover, at the initial crack angles beyond the occurrence of pure mode II, where the stress intensity factor of mode I becomes negative, this criterion is not applicable. Therefore, in this research work, the IMTS criterion which has been implemented in the extended finite element method and is applicable under tensile and compressive loading conditions has been utilized to examine the crack propagation path in SCB and CBD specimens. Also, in order to evaluate the Improved MTS criterion in determination of the crack propagation path at angles beyond the occurrence of pure mode II, a laboratory experimental study on the CBD specimen has been conducted.

When the IMTS criterion is employed in simulation of the crack propagation path in the SCB specimen, the results are well matched with modeling results by the GMTS criterion and also laboratory experimental test results. In the IMTS criterion, the crack initiation angle at the initial crack angle of $60^{\circ}$ could be calculated, whereas due to the negative stress intensity factor of mode I at this angle, the MTS and GMTS criteria are not applicable.

The estimation of crack propagation in the CBD specimen using the IMTS criterion shows a good agreement with the experimental results. Also it is observed that unlike MTS and GMTS criteria, the IMTS criterion is applicable in this specimen at initial crack angles beyond the occurrence of pure mode II. The results demonstrate that using the MTS criterion in simulating crack propagation in both SCB and CBD specimens introduces an unknown degree of uncertainty into the results. The MTS criterion underestimates the crack initiation angle in the SCB specimen and overestimates it in the CBD specimen compared with the laboratory experimental and also simulation results by the IMTS criterion. The results of the IMTS criterion show that it is well matched with the experimental results and can be used in different specimens under different tensile and compressive loading conditions.

\section{References}

1. Al-Shayea N.A., 2005, Crack propagation trajectories for rocks under mixed mode I-II fracture, Engineering Geology, 81, 84-97

2. Aliha M., Ayatollahi M., Smith D., Pavier M., 2010, Geometry and size effects on fracture trajectory in a limestone rock under mixed mode loading, Engineering Fracture Mechanics, 77, 2200-2212

3. Atkinson C., Smelser R., Sanchez J., 1982, Combined mode fracture via the cracked Brazilian disk test, International Journal of Fracture, 18, 279-291

4. Awaji H., Sato S., 1978, Combined mode fracture toughness measurement by the disk test, Journal of Engineering Materials and Technology, 100, 175-182

5. Ayatollahi M., Aliha M., 2008, On the use of Brazilian disc specimen for calculating mixed mode I-II fracture toughness of rock materials, Engineering Fracture Mechanics, 75, 4631-4641

6. Babanouri N., Nasab S.K., Baghbanan A., Mohamadi H.R., 2011, Over-consolidation effect on shear behavior of rock joints, International Journal of Rock Mechanics and Mining Sciences, 48, 1283-1291

7. Belytschko T., Black T., 1999, Elastic crack growth in finite elements with minimal remeshing, International Journal for Numerical Methods in Engineering, 45, 601-620

8. Bobet A., 1997, Fracture Coalescence in Rock Materials: Experimental Observations and Numerical Predictions, Massachusetts Institute of Technology

9. Bobet A., Einstein H.H., 1998, Numerical modeling of fracture coalescence in a model rock material, International Journal of Fracture, 92, 221-252 
10. Budyn E., Zi G., Mö̈s N., Belytschko T., 2004, A method for multiple crack growth in brittle materials without remeshing, International Journal for Numerical Methods in Engineering, 61, 1741-1770

11. Campilho R., Banea M., Chaves F., Da Silva L., 2011, Extended Finite Element Method for fracture characterization of adhesive joints in pure mode I, Computational Materials Science, 50, $1543-1549$

12. Chen C.-S., Pan E., Amadei B., 1998, Fracture mechanics analysis of cracked discs of anisotropic rock using the boundary element method, International Journal of Rock Mechanics and Mining Sciences, 35, 195-218

13. Choi S., Haberfield C., Johnston I., 1988, Determining the tensile strength of soft rock, Geotechnical Engineering, 19

14. Chong K.P., Kuruppu M.D., Kuszmaul J.S., 1987, Fracture toughness determination of layered materials, Engineering Fracture Mechanics, 28, 43-54

15. Dolbow J.E., 1999, An Extended Finite Element Method with Discontinuous Enrichment for Applied Mechanics, Northwestern University

16. Eftekhari M., Baghbanan A., Hashemolhosseini H., 2014, Determining stress intensity factor for Cracked Brazilian Disc using extended Finite Element Method, International Journal of Engineering Science, 3, 890-893

17. Eftekhari M., Baghbanan A., Hashemolhosseini H., 2015a, Extended finite element simulation of crack propagation in Cracked Brazilian Disc, Journal of Mining and Environment, 6, 95-102

18. Eftekhari, M., Baghbanan, A., Hashemolhosseini, H., 2015b, Fracture propagation in a cracked semicircular bend specimen under mixed mode loading using extended finite element method, Arabian Journal of Geosciences, 8, 9635-9646

19. Eftekhari M., Baghbanan A., Hashemolhosseini H., 2016, Crack propagation in rock specimen under compressive loading using extended finite element method, Arabian Journal of Geosciences, 9, 1-10

20. Eftekhari M., Baghbanan A., Hashemolhosseini H., Amrollahi H., 2015c, Mechanism of fracture in macro-and micro-scales in hollow centre cracked disc specimen, Journal of Central South University of Technology, 22, 4426-4433

21. Erdogan F., Sin G., 1963, On the crack extension in plates under plane loading and transverse shear, Journal of Basic Engineering, 85, 519

22. FAn C., Jing X.Q., 2013, Numerical study of crack propagation path in three-point bending beam using Extended Finite Element Method, Applied Mechanics and Materials, 353-356, 3615-3618

23. Fowell R., 1995, Suggested method for determining mode I fracture toughness using cracked chevron notched Brazilian disc (CCNBD) specimens, International Journal of Rock Mechanics and Mining Sciences and Geomechanics Abstracts, 57-64

24. Ghazvinian A., Nejati H.R., Sarfarazi V., Hadei M.R., 2013, Mixed mode crack propagation in low brittle rock-like materials, Arabian Journal of Geosciences, 6, 4435-4444

25. Grassl P., Rempling R., 2007, Influence of volumetric-deviatoric coupling on crack prediction in concrete fracture tests, Engineering Fracture Mechanics, 74, 1683-1693

26. Haeri H., Shahriar K., Marji M.F., Moarefvand P., 2014, Cracks coalescence mechanism and cracks propagation paths in rock-like specimens containing pre-existing random cracks under compression, Journal of Central South University of Technology, 21, 2404-2414

27. Hussain M., Pu S., Underwood J., 1974, Strain energy release rate for a crack under combined mode I and mode II, Fracture Analysis, 1, 560

28. Huynh D., Belytschko T., 2009, The extended finite element method for fracture in composite materials, International Journal for Numerical Methods in Engineering, 77, 214-239

29. IsAKsSOn P., StÅHLe P., 2002, Mode II crack paths under compression in brittle solids - a theory and experimental comparison, International Journal of Solids and Structures, 39, 2281-2297 
30. Kuruppu M., Obara Y., Ayatollahi M., Chong K., Funatsu T., 2015, ISRM-suggested method for determining the mode I static fracture toughness using semi-circular bend specimen, The ISRM Suggested Methods for Rock Characterization, Testing and Monitoring, 2007-2014, Springer, 107-114

31. Kuruppu M.D., Chong K.P., 2012, Fracture toughness testing of brittle materials using semicircular bend (SCB) specimen, Engineering Fracture Mechanics, 91, 133-150

32. Lin Q., Fakhimi A., Haggerty M., Labuz J., 2009, Initiation of tensile and mixed-mode fracture in sandstone, International Journal of Rock Mechanics and Mining Sciences, 46, 489-497

33. Liu H., Kou S., Lindqvist P.-A., TAng C., 2007, Numerical modelling of the heterogeneous rock fracture process using various test techniques, Rock Mechanics and Rock Engineering, 40, $107-144$

34. Mergheim J., Kuhl E., Steinmann P., 2005, A finite element method for the computational modelling of cohesive cracks, International Journal for Numerical Methods in Engineering, 63, 276-289

35. Mö̈s N., Dolbow J., Belytschko T., 1999, A finite element method for crack growth without remeshing, International Journal for Numerical Methods in Engineering, 46, 131-150

36. Nuismer R., 1975, An energy release rate criterion for mixed mode fracture, International Journal of Fracture, 11, 245-250

37. Palaniswamy K., Knauss W., 1972, Propagation of a crack under general, in-plane tension, International Journal of Fracture, 8, 114-117

38. Sharafisafa M., Nazem M., 2014, Application of the distinct element method and the extended finite element method in modelling cracks and coalescence in brittle materials, Computational Materials Science, 91, 102-121

39. Shen B., Stephansson O., 1994, Modification of the G-criterion for crack propagation subjected to compression, Engineering Fracture Mechanics, 47, 177-189

40. Shiryaev A., Kotkis A., 1983. Methods for determining fracture toughness of brittle porous materials, Industrial Laboratory, 48, 917-918

41. SiH G.C., 1974, Strain-energy-density factor applied to mixed mode crack problems, International Journal of Fracture, 10, 305-321

42. Singh I., Mishra B., Bhattacharya S., Patil R., 2012, The numerical simulation of fatigue crack growth using extended finite element method, International Journal of Fatigue, 36, 109-119

43. Song L., Huang S., YAng S., 2004, Experimental investigation on criterion of three-dimensional mixed-mode fracture for concrete, Cement and Concrete Research, 34, 913-916

44. Theocaris P., Andrianopoulos N., 1982, A modified strain-energy density criterion applied to crack propagation, Journal of Applied Mechanics, 49, 81-86

45. Thiercelin M., 1989, Fracture toughness and hydraulic fracturing, International Journal of Rock Mechanics and Mining Sciences and Geomechanics Abstracts, 177-183

46. Thiercelin, M., Roegiers, J., 1986, Fracture toughness determination with the modified ring test, International Symposium on Engineering in Complex rock Formations, Beijing, China, 1-8

47. Wang Q., Jia X., Kou S., Zhang Z., Lindqvist P.-A., 2004, The flattened Brazilian disc specimen used for testing elastic modulus, tensile strength and fracture toughness of brittle rocks: analytical and numerical results, International Journal of Rock Mechanics and Mining Sciences, 41, 245-253

48. WANG Q.-Z., XING L., 1999, Determination of fracture toughness K IC by using the flattened Brazilian disk specimen for rocks, Engineering Fracture Mechanics, 64, 193-201

49. Wu Z., Wong L.N.Y., 2012, Frictional crack initiation and propagation analysis using the numerical manifold method, Computers and Geotechnics, 39, 38-53

50. Xeidakis G., Samaras I., Zacharopoulos D., Papakaliatakis G., 1997, Trajectories of unstably growing cracks in mixed mode I-II loading of marble beams, Rock Mechanics and Rock Engineering, 30, 19-33 